\title{
Mean-field description of collapsing and exploding Bose-Einstein condensates
}

\author{
Sadhan K. Adhikari \\ Instituto de Física Teórica, Universidade Estadual Paulista, 01.405-900 São Paulo, São Paulo, Brazil
}

(Received 4 February 2002; revised manuscript received 21 March 2002; published 19 July 2002)

\begin{abstract}
We perform numerical simulations based on the time-dependent mean-field Gross-Pitaevskii equation to understand some aspects of a recent experiment by Donley et al. [Nature (London) 412, 295 (2001)] on the dynamics of collapsing and exploding Bose-Einstein condensates of ${ }^{85} \mathrm{Rb}$ atoms. These authors manipulated the atomic interaction by an external magnetic field via a Feshbach resonance, thus changing the repulsive condensate into an attractive one, and vice versa. In the actual experiment they suddenly changed the scattering length of atomic interaction from a positive to a large negative value on a preformed condensate in an axially symmetric trap. Consequently, the condensate collapsed and ejected atoms via explosion. We find that the present mean-field analysis can explain some aspects of the dynamics of the collapsing and exploding BoseEinstein condensates.
\end{abstract}

DOI: 10.1103/PhysRevA.66.013611

PACS number(s): 03.75.Fi

\section{INTRODUCTION}

Recent successful detection [1-3] of Bose-Einstein condensates (BEC's) in dilute bosonic atoms employing magnetic traps at ultralow temperatures has intensified experimental activities on various aspects of the condensate. On the theoretical front, numerical simulation based on the timedependent nonlinear mean-field Gross-Pitaevskii (GP) equation [4] has provided a satisfactory account of some of these experiments [5-9]. Since the detection of BEC's for ${ }^{7} \mathrm{Li}$ atoms with attractive interaction, one problem of extreme interest is the dynamical study of the formation and decay of BEC's for attractive atomic interactions [3].

For attractive interaction the condensate is stable for a maximum critical number $N_{\text {cr }}$ of atoms [3]. Measurements of $N_{\text {cr }}[3,10]$ are in reasonable agreement with mean-field analyses for BEC's of ${ }^{7} \mathrm{Li}$ in a spherically symmetric trap $[5,11]$, although there is some discrepancy for BEC's of ${ }^{85} \mathrm{Rb}$ in an axially symmetric trap $[12,13]$. If the number of atoms can somehow be increased beyond this critical number, the condensate collapses due to interatomic attraction, emitting atoms until the number of atoms is reduced below $N_{\mathrm{cr}}$ and a stable configuration is reached. With a supply of atoms from an external source the condensate can grow again and thus a series of collapses can take place, which has been observed experimentally in a BEC of ${ }^{7} \mathrm{Li}$ with attractive interaction [3]. A theoretical mean-field analysis has been able to explain this dynamics [5-9].

Recently, a more challenging experiment was performed by Donley et al. [14] on an attractive condensate of ${ }^{85} \mathrm{Rb}$ atoms [10] in an axially symmetric trap, where they manipulated the interatomic interaction by changing the external magnetic field, exploiting a nearby Feshbach resonance [15]. In the vicinity of a Feshbach resonance the atomic scattering length $a$ can be varied over a huge range by adjusting the external magnetic field. Consequently, they were able to suddenly change the atomic scattering length by a large amount for a BEC's of ${ }^{85} \mathrm{Rb}$ atoms [10]. They even changed the sign of the scattering length, thus transforming a repulsive condensate into an attractive one. The original experiment on attractive ${ }^{7} \mathrm{Li}$ atoms [3] did not use a Feshbach resonance; hence the atomic interaction was fixed. This restricts the number of atoms in the ${ }^{7} \mathrm{Li} \mathrm{BEC}$ to a number close to $N_{\text {cr }}$ and the collapse is driven by a stochastic process $[3,14]$.

In the experiment conducted at JILA, Donley et al. changed a stable preformed repulsive condensate of ${ }^{85} \mathrm{Rb}$ atoms into a highly explosive and collapsing attractive condensate and studied the dynamics of collapsing and exploding condensates [14]. The natural scattering length of ${ }^{85} \mathrm{Rb}$ atoms is negative (attractive). By exploiting the Feshbach resonance Donley et al. made it positive (repulsive) in the initial state, where the number of atoms, unlike in the experiment with ${ }^{7} \mathrm{Li}$ [3], could be arbitrarily large. So immediately after the jump in the scattering length to a large negative value, one has a highly unstable BEC, where the number of atoms could be much larger than $N_{\mathrm{cr}}$. Donley et al. have provided a quantitative estimate of the explosion of this BEC by measuring the number of atoms remaining in the condensate as a function of time until an equilibrium is reached. They claim that their experiment reveals many interesting phenomena that challenge theoretical models. The fundamental physical process underlying the explosion remains a mystery.

In this paper we perform a mean-field analysis based on the time-dependent GP equation to understand some aspects of the above collapse and explosion of an attractive condensate of ${ }^{85} \mathrm{Rb}$ atoms in an axially symmetric trap. To account for the loss of atoms from the strongly attractive condensate we include an absorptive nonlinear three-body recombination term in the GP equation. Three-body recombination leads to the formation of diatomic molecules with liberation of the energy responsible for energetic explosion with ejection of matter from the BEC. This process could be termed "atomic fusion," in contrast to nuclear fusion in stars. The three-body recombination rate we use in numerical simulations is in agreement with previous experimental measurement [16] and theoretical calculation [17]. The numerical method we use for the solution of the time-dependent GP equation with an axially symmetric trap has appeared elsewhere $[13,18,19]$. We find that the present mean-field numerical simulation provides a fair description of some features of the experiment of Donley et al. [14]. 
There have been other theoretical studies based on the mean-field GP equation [6-9] to deal with dynamical collapse including an absorptive term to account for the loss of particles. In most cases the loss mechanism is three-body recombination as in the present study. Duine and Stoof [7] proposed that the loss arises due to a new elastic process. Instead of attempting a full numerical solution of the GP equation with axial symmetry, these investigations used various approximations to study the time evolution of the condensate or employed a spherically symmetric trap. Duine and Stoof [7] considered the full anisotropic dynamics, but used a Gaussian approximation for the wave function rather than an exact numerical solution. Most of the other studies employed a spherically symmetric trap $[6,8]$. However, the investigation of Ref. [9] employed an axially symmetric trap to describe some aspects of the experiment of Donley et al. and we comment on this work in Sec. IV. In the present investigation we consider the complete numerical solution of the mean-field GP equation for an axially symmetric trap as in the experiment of Donley et al. It is realized that an approximate solution as in the previous studies cannot explain the dynamics of this experiment $[9,14]$.

In Sec. II we present the theoretical model and the numerical method for its solution. In Sec. III we present our results, which we compare with the experiment of Donley et al. Finally, in Secs. IV and V we present a brief discussion and concluding remarks.

\section{NONLINEAR GROSS-PITAEVSKII EQUATION}

\section{A. Theoretical model equations}

The time-dependent Bose-Einstein condensate wave function $\Psi(\mathbf{r} ; \tau)$ at position $\mathbf{r}$ and time $\tau$ allowing for atomic loss may be described by the following mean-field nonlinear GP equation $[4,5]$ :

$$
\begin{aligned}
& {\left[-i \hbar \frac{\partial}{\partial \tau}-\frac{\hbar^{2} \nabla^{2}}{2 m}+V(\mathbf{r})+g N|\Psi(\mathbf{r} ; \tau)|^{2}-\frac{i \hbar}{2}\right.} \\
& \left.\quad \times\left[K_{2} N|\Psi(\mathbf{r} ; \tau)|^{2}+K_{3} N^{2}|\Psi(\mathbf{r} ; \tau)|^{4}\right]\right] \Psi(\mathbf{r} ; \tau)=0 .
\end{aligned}
$$

Here $m$ is the mass and $N$ the number of atoms in the condensate; $g=4 \pi \hbar^{2} a / m$ is the strength of interatomic interaction, with $a$ the atomic scattering length. A positive $a$ corresponds to a repulsive interaction and a negative $a$ to an attractive interaction. The terms $K_{2}$ and $K_{3}$ denote two-body dipolar and three-body recombination loss-rate coefficients, respectively. There are many ways to account for the loss mechanism $[6,7]$. It is quite impossible to include them all in a self-consistent fashion. Here we simulate the atom loss via the most important quintic three-body term $K_{3}[6,8,9]$. The contribution of the cubic two-body loss term [16] is expected to be negligible $[6,9]$ compared to the three-body term in the present problem of the collapsed condensate with large density, and will not be considered here.

The trap potential with cylindrical symmetry may be written as $V(\mathbf{r})=\frac{1}{2} m \omega^{2}\left(r^{2}+\lambda^{2} z^{2}\right)$ where $\omega$ is the angular fre- quency in the radial direction $r$ and $\lambda \omega$ that in the axial direction $z$. We are using the cylindrical coordinate system $\mathbf{r} \equiv(r, \theta, z)$ with $\theta$ the azimuthal angle. The normalization condition of the wave function is $\int d \mathbf{r}|\Psi(\mathbf{r} ; \tau)|^{2}=1$.

In the absence of angular momentum the wave function has the form $\Psi(\mathbf{r} ; \tau)=\psi(r, z ; \tau)$. Now transforming to dimensionless variables defined by $x=\sqrt{2} r / l, y=\sqrt{2} z / l, t$ $=\tau \omega, l \equiv \sqrt{\hbar /(m \omega)}$, and

$$
\phi(x, y ; t) \equiv \frac{\varphi(x, y ; t)}{x}=\sqrt{\frac{l^{3}}{\sqrt{8}}} \psi(r, z ; \tau),
$$

we get

$$
\begin{gathered}
{\left[-i \frac{\partial}{\partial t}-\frac{\partial^{2}}{\partial x^{2}}+\frac{1}{x} \frac{\partial}{\partial x}-\frac{\partial^{2}}{\partial y^{2}}+\frac{1}{4}\left(x^{2}+\lambda^{2} y^{2}-\frac{4}{x^{2}}\right)\right.} \\
+8 \sqrt{2} \pi n\left|\frac{\varphi(x, y ; t)}{x}\right|^{2} \\
\left.-i \xi n^{2}\left|\frac{\varphi(x, y ; t)}{x}\right|^{4}\right] \varphi(x, y ; t)=0,
\end{gathered}
$$

where $n=N a / l$ and $\xi=4 K_{3} /\left(a^{2} l^{4} \omega\right)$. This scaled meanfield equation has the correct $n$ dependence of the three-body term so that the same equation can be used to study the decay rate of different initial and final scattering lengths $a_{\text {initial }}$ and $a_{\text {collapse }}$, respectively, and initial number of atoms $N_{0}$. In this study the term $K_{3}$ will be used for the description of atom loss in the case of attractive interaction, where the scattering length $a$ is negative. From theoretical [20] and experimental [16] studies it has been found that for negative $a K_{3}$ increases rapidly as $|a|^{n}$, where the theoretical study [20] favors $n=2$ for smaller values of $|a|$. For larger $|a|$, a much larger rate of increase may take place $[17,20]$. There are experimental [21] and theoretical $[17,20,22]$ estimates of $K_{3}$ for ${ }^{87} \mathrm{Rb},{ }^{23} \mathrm{Na}$, and ${ }^{7} \mathrm{Li}$ away from Feshbach resonances. However, no thorough and systematic study of the variation of $K_{3}$ near a Feshbach resonance has been performed [23]. An accurate representation of the variation of $K_{3}$ of ${ }^{85} \mathrm{Rb}$ near the Feshbach resonance is beyond the scope of this study and here we represent this variation via a quadratic dependence: $K_{3} \sim a^{2}$. This makes the parameter $\xi$ above a constant for an experimental setup with fixed $l$ and $\omega$, and in the present study we use a constant $\xi$.

The normalization condition of the wave function becomes

$$
\mathcal{N}_{\text {norm }} \equiv 2 \pi \int_{0}^{\infty} d x \int_{-\infty}^{\infty} d y|\varphi(x, y ; t)|^{2} x^{-1}=1
$$

For $K_{3}=0, \mathcal{N}_{\text {norm }}=1$; however, in the presence of loss $K_{3}$ $>0, \mathcal{N}_{\text {norm }}<1$. The number of remaining atoms $N$ in the condensate is given by $N=N_{0} \mathcal{N}_{\text {norm }}$, where $N_{0}$ is the initial number.

The root mean square (rms) sizes $x_{\text {rms }}$ and $y_{\text {rms }}$ are defined by 


$$
\begin{gathered}
x_{\mathrm{rms}}^{2}=\mathcal{N}_{\mathrm{norm}}^{-1} 2 \pi \int_{0}^{\infty} d x \int_{-\infty}^{\infty} d y|\varphi(x, y ; t)|^{2} x, \\
y_{\mathrm{rms}}^{2}=\mathcal{N}_{\mathrm{norm}}^{-1} 2 \pi \int_{0}^{\infty} d x \int_{-\infty}^{\infty} d y|\varphi(x, y ; t)|^{2} y^{2} x^{-1} .
\end{gathered}
$$

\section{B. Numerical details}

We solve the GP equation (2.3) numerically using a timeiteration method elaborated in Refs. $[13,18,19,24]$. The full GP Hamiltonian is conveniently broken into three parts$H_{x}, H_{y}$, and $H_{n}$ - the first containing the $x$-dependent derivative terms, the second containing the $y$-dependent derivative terms, and the third containing the remaining linear and nonlinear terms. The GP equations for the first two parts are defined on a two-dimensional set of grid points $N_{x} \times N_{y}$ using the Crank-Nicholson discretization method. The resultant tridiagonal equations along $x$ and $y$ directions are solved alternately by the Gaussian elimination method along the $x$ and $y$ directions [24]. The GP equation for the third part does not contain any space derivative and is solved essentially exactly. Effectively, each time iteration of the GP equation is broken up into three parts-using $H_{x}, H_{y}$ and $H_{n}$. For a small time step $\Delta$ the error involved in this break-up procedure along the $x$ and $y$ directions is quadratic in $\Delta$ and hence can be neglected. For numerical purposes we discretize the GP equation using the time step $\Delta=0.001$ and space step 0.1 for both $x$ and $y$ spanning $x$ from 0 to 15 and $y$, from -30 to 30. This domain of space was sufficient to encompass the whole condensate wave function even during and after collapse and explosion. The preparation of the initial repulsive wave function is now a routine job and was done by increasing the nonlinearity $n$ of the GP equation (2.3) by 0.0001 in each time step $\Delta$ during time iteration, starting with the known harmonic oscillator solution of Eq. (2.3) for $n=\xi$ $=0[13]$.

It is now appropriate to calculate the parameters of the present dimensionless GP equation (2.3) corresponding to the experiment of Donley et al. We follow the notation and nomenclature of Ref. [14]. Their radial and axial trap frequencies are $\nu_{\text {radial }}=17.5 \mathrm{~Hz}$ and $\nu_{\text {axial }}=6.8 \mathrm{~Hz}$, respectively, leading to $\lambda=0.389$. The harmonic oscillator length $l$ of ${ }^{85} \mathrm{Rb}$ atoms for $\omega=2 \pi \times 17.5 \mathrm{~Hz}$ and $m \approx 79176 \mathrm{MeV}$ is $l=\sqrt{\hbar /(m \omega)}=26070 \AA$. One unit of time $t$ of Eq. (2.3) is $1 / \omega$ or $0.009095 \mathrm{~s}$. Donley et al. prepared a stable ${ }^{85} \mathrm{Rb}$ condensate of $N_{0}=16000$ atoms with scattering length $a_{\text {initial }}=7 a_{0}, a_{0}=0.5292 \AA$, such that the initial $n=2.274$. Then during an interval of time $0.1 \mathrm{~ms}$ the scattering length was ramped to $a=a_{\text {collapse }}=-30 a_{0}$ such that the final $n=$ -9.744. The final condensate is strongly attractive and unstable and undergoes a sequence of collapse and explosion.

The initial value of $n(=2.274)$ was attained after 22740 time steps. The nonlinearity $n$ is then ramped from 2.274 to -9.744 in $0.1 \mathrm{~ms}$. As one unit of dimensionless time $t$ is $0.009095 \mathrm{~s}, 0.1 \mathrm{~ms}$ corresponds to 11 steps of time $\Delta$. In the present simulation, $n$ was ramped from 2.274 to -9.744 in the GP equation by equal amounts in 11 steps. The absorptive term $\xi$ was set equal to zero during the above time

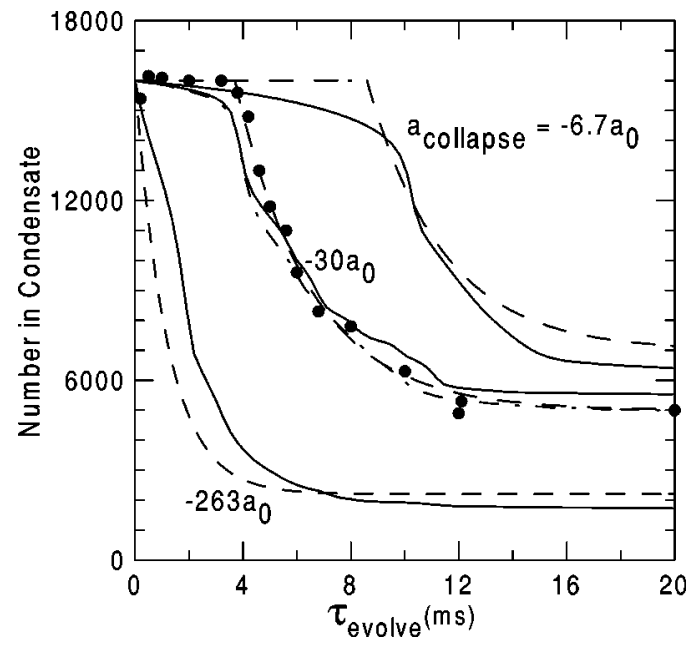

FIG. 1. Number of remaining atoms in the condensate of $16000{ }^{85} \mathrm{Rb}$ atoms after ramping the scattering length from $a_{\text {initial }}$ $=7 a_{0}$ to $a_{\text {collapse }}=-6.7 a_{0},-30 a_{0}$, and $-263 a_{0}$ in $0.1 \mathrm{~ms}$ as a function of evolution time $\tau_{\text {evolve }}$ in ms. Solid circles, experiment for $a_{\text {collapse }}=-30 a_{0}$ [14]; full line, theory $(\xi=2)$; dash-dotted line, theory $\left(\xi=3, a_{\text {collapse }}=-30 a_{0}\right)$; dashed line, average over preliminary, unanalyzed data using Eq. (3.1) [25].

iteration. Now the system is prepared for simulation of the collapse and explosion.

For the simulation of the collapse and explosion the cubic nonlinear term was maintained constant and a nonzero value of $\xi$ was chosen. The time evolution of the GP equation is continued as a function of time $t=\tau_{\text {evolve }}$ starting at 0 . The time evolution was continued using a time step $\Delta=0.001$. After a small experimentation it was found that $\xi=2$ fits the experiment of Donley et al. satisfactorily. Unless otherwise specified, this value of $\xi$ was used in all simulations reported in this paper for different $a_{\text {initial }}, a_{\text {collapse }}$, and $N_{0}$.

It is useful to compare this value of $\xi(=2)$ with the experimental [16] and theoretical [20] estimates of the threebody loss rate of ${ }^{85} \mathrm{Rb}$. For this we recall that $K_{3}$ $=\xi a^{2} l^{4} \omega / 4$. Under the experimental condition of an external magnetic field of $250 \mathrm{G}$ on ${ }^{85} \mathrm{Rb}$ [16], the scattering length was $a \sim-370 a_{0}$. Consequently, the present value of $\xi$ (=2) corresponds to $K_{3} \simeq 9 \times 10^{-25} \mathrm{~cm}^{6} / \mathrm{s}$ for $a \sim-370 a_{0}$, which is about the experimental rate $K_{3}=\left(4.24_{-0.29}^{+0.70} \pm 0.85\right)$ $\times 10^{-25} \mathrm{~cm}^{6} / \mathrm{s}[16]$ and about 1.3 times the theoretical rate $K_{3}=6.7 \times 10^{-25} \mathrm{~cm}^{6} / \mathrm{s}$ at $a \sim-370 a_{0}[17]$.

\section{NUMERICAL RESULTS}

The numerical simulation using Eq. (2.3) with a nonzero $\xi$ immediately yields the remaining number of atoms in the condensate after the jump in scattering length. The remaining number of atoms vs time is plotted in Fig. 1 for $a_{\text {initial }}$ $=7 a_{0}, a_{\text {collapse }}=-30 a_{0}, \xi=2$, and $N_{0}=16000$, and compared with the experimental data. In this figure we also plot the result in this case for $\xi=3$, which leads to a better agreement with experiment for this specific case. However, the use of $\xi=2$ leads to a more satisfactory overall agreement with experiment. Except for this single curve in Fig. 1 and the plot in Fig. 4(a) below, which are calculated with $\xi=3$, all results 
reported in this paper are calculated with $\xi=2$.

In the experiment of Donley et al. [14] it was observed that the strongly attractive condensate remains stable after preparation with a constant number of atoms for an interval of time $t_{\text {collapse }}$, called the collapse time. This behavior is physically expected. Immediately after the jump in scattering length from $7 a_{0}$ to $-30 a_{0}$, the attractive condensate shrinks in size during $t_{\text {collapse }}$, until the central density increases to a maximum. Then the absorptive three-body term takes full control to initiate the explosion. Consequently, the number of atoms remains constant for $\tau_{\text {evolve }}<t_{\text {collapse }}$. The present result (full line) also shows a similar behavior. However, in this simulation the absorptive term is operative from $\tau_{\text {evolve }}$ $=0$ and the atom number decreases right from the beginning, albeit at a much smaller rate for $\tau_{\text {evolve }}<t_{\text {collapse }}$.

Donley et al. repeated their experiment with different values of $a_{\text {initial }}, a_{\text {collapse }}$, and $N_{0}$ [14]. For $a_{\text {initial }}=7 a_{0}$ we repeated our calculation with the following values of final scattering length: $a_{\text {collapse }}=-263 a_{0}$ and $-6.7 a_{0}$. These results are also plotted in Fig. 1 and agree with the unpublished, preliminary unanalyzed data [25]. The initial delay $t_{\text {collapse }}$ in starting the explosion is large for small $\left|a_{\text {collapse }}\right|$ as we see in Fig. 1. A similar effect was observed in the experiment for an initial condensate of 6000 atoms as shown in Fig. 2 of [14]. After a sequence of collapse and explosion, Donley et al. observed a "remnant" condensate of $N_{\text {remnant }}$ atoms at large times containing a certain constant fraction of the initial $N_{0}$ atoms. Figure 1 shows such a behavior.

The above evolution of the condensate after the jump in scattering length to $-30 a_{0}$ from $7 a_{0}$ for $N_{0}=16000$ can be understood from a study of the wave function and we display the central part of the wave function in Fig. 2 for $\tau_{\text {evolve }}$ $=0,3.6,3.8$, and $8 \mathrm{~ms}$. The wave function immediately after the jump at time $\tau_{\text {evolve }}=0$ is essentially the same as that before the jump at $-0.1 \mathrm{~ms}$. There is not enough time for the wave function to be modified at $\tau_{\text {evolve }}=0$. From Fig. 2 we find that at $3.6 \mathrm{~ms}$ the wave function is only slightly narrower than at $0 \mathrm{~ms}$ but still smooth and has not yet collapsed sufficiently. As $\tau_{\text {evolve }}$ increases, the wave function contracts further and the explosion starts. At $3.8 \mathrm{~ms}$ some spikes (irregularities) have appeared in the wave function showing, the beginning of the explosion and loss. From the study of the wave functions we find that the explosion starts at $\tau_{\text {evolve }}=t_{\text {collapse }} \simeq 3.7 \mathrm{~ms}$, in agreement with the experiment of Donley et al. We also find that at $3.7 \mathrm{~ms}$ before the loss began the bulk BEC did not contract dramatically, as also observed in the experiment. In the numerical simulation for this case we find that at $\tau_{\text {evolve }}=0, x_{\text {rms }}=2.98 \mu \mathrm{m}$ and $y_{\mathrm{rms}}=4.21 \mu \mathrm{m}$, and at $\tau_{\text {evolve }}=3.7 \mathrm{~ms}, x_{\mathrm{rms}}=2.53 \mu \mathrm{m}$, and $y_{\mathrm{rms}}=4.10 \mu \mathrm{m}$. From Fig. 2 we see that at $8 \mathrm{~ms}$ the wave function is very spiky, corresponding to the violent ongoing explosion.

Donley et al. fitted the decay in the number of atoms during particle loss to a decay constant $\tau_{\text {decay }}$ via the formula

$$
\begin{aligned}
N\left(\tau_{\text {evolve }}\right)= & N_{\text {remnant }}+\left(N_{0}-N_{\text {remnant }}\right) \\
& \times e^{\left(t_{\text {collapse }}-\tau_{\text {evolve }}\right) / \tau_{\text {decay }}}
\end{aligned}
$$

for $\tau_{\text {evolve }}>t_{\text {collapse }}$. In Fig. 1 we also plot $N\left(\tau_{\text {evolve }}\right)$ of Eq.
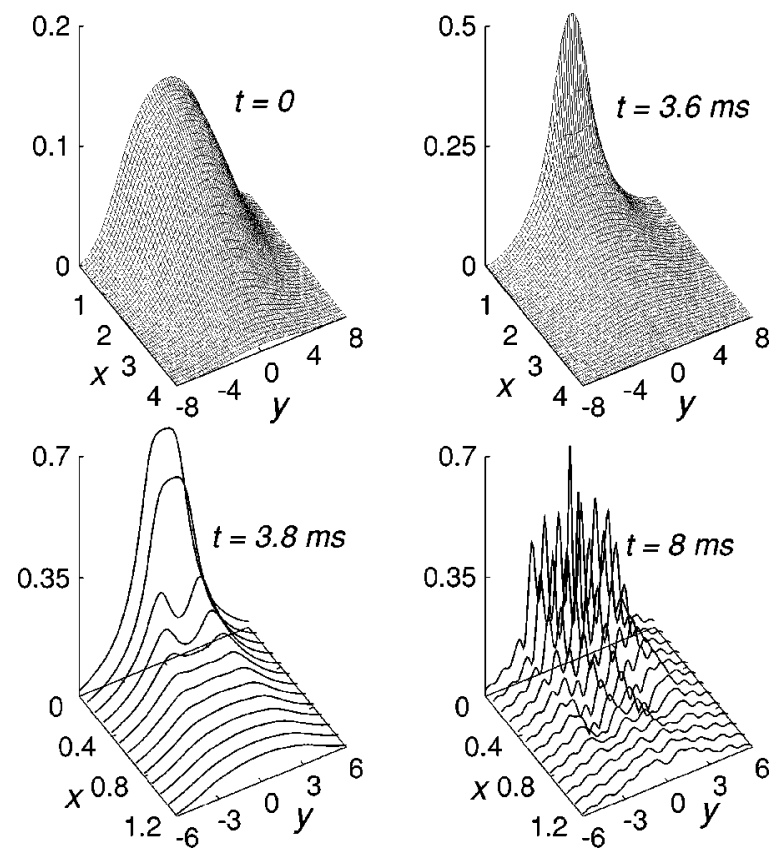

FIG. 2. The central part of the dimensionless wave function $|\phi(x, y)| \equiv|\varphi(x, y) / x|$ of the condensate on a $0.1 \times 0.1$ grid for $\xi$ $=2$ after the jump in the scattering length of a BEC of $16000{ }^{85} \mathrm{Rb}$ atoms from $a_{\text {initial }}=7 a_{0}$ to $a_{\text {collapse }}=-30 a_{0}$ at times $\tau_{\text {evolve }}=0,3.6$ $\mathrm{ms}, 3.8 \mathrm{~ms}$, and $8 \mathrm{~ms}$. The quantities $x$ and $y$ are expressed in units of $l / \sqrt{2}$, where $l=26070 \AA$.

(3.1) (dashed line) for $a_{\text {collapse }}=-263 a_{0},-30 a_{0}$, and $-6.7 a_{0}$ with respective decay rates $\tau_{\text {decay }}=1.2 \mathrm{~ms}, 2.8 \mathrm{~ms}$, and $2.8 \mathrm{~ms}$ [25]. For a wide variation of parameters $a_{\text {initial }}, a_{\text {collapse }}$, and $N_{0}, \tau_{\text {decay }}$ varies approximately between 1 and 3 . The results of the present simulation (full line) agree well with the average experimental result of Eq. (3.1) for three different $a_{\text {collapse }}$ (dashed line) [25].

Next we repeated our calculation for several other values of $a_{\text {initial }}, a_{\text {collapse }}$, and $N_{0}$. These results are plotted in Fig. 3 for (a) $a_{\text {initial }}=89 a_{0}, a_{\text {collapse }}=-15 a_{0}$, and $N_{0}=6000$, (b) $a_{\text {initial }}=0.64 a_{0}, \quad a_{\text {collapse }}=-6.6 a_{0}, \quad$ and $N_{0}=14500$, (c) $a_{\text {initial }}=0.64 a_{0}, a_{\text {collapse }}=-6.6 a_{0}$, and $N_{0}=5500$, and (d) $a_{\text {initial }}=7 a_{0}, a_{\text {collapse }}=-263 a_{0}$, and $N_{0}=6000$. The agreement of the result of simulation with unpublished, preliminary unanalyzed data is good in all four cases reported in Fig. 3 [25].

The decay curves in Fig. 3 are different, although they have certain general features that determine the decay constant $\tau_{\text {decay }}$, collapse time $t_{\text {collapse }}$, and number of atoms in the remnant. Experimentally, the fraction of atoms that went into the remnant decreased with increasing $\left|a_{\text {collapse }}\right|$ and was $\sim 40 \%$ for $\left|a_{\text {collapse }}\right|<10 a_{0}$ and $\sim 10 \%$ for $\left|a_{\text {collapse }}\right|$ $>100 a_{0}$. Figures 1 and 3 also show this behavior. The values of $\tau_{\text {decay }}$ for plots in Figs. 3(a)-3(d) are $1.5 \mathrm{~ms}, 2.4 \mathrm{~ms}, 3.3$ $\mathrm{ms}$, and $1.9 \mathrm{~ms}$, respectively, lying in the range $\sim 1-3 \mathrm{~ms}$ [25]. The general features in the behavior of remnant number and collapse time are discussed in the following.

Donley et al. provided a quantitative measurement of the variation of collapse time $t_{\text {collapse }}$ with the final scattering length $a_{\text {collapse }}$ for a given $a_{\text {initial }}=0$ and $N_{0}=6000$. We also calculated this variation using our model given by Eq. (2.3). 

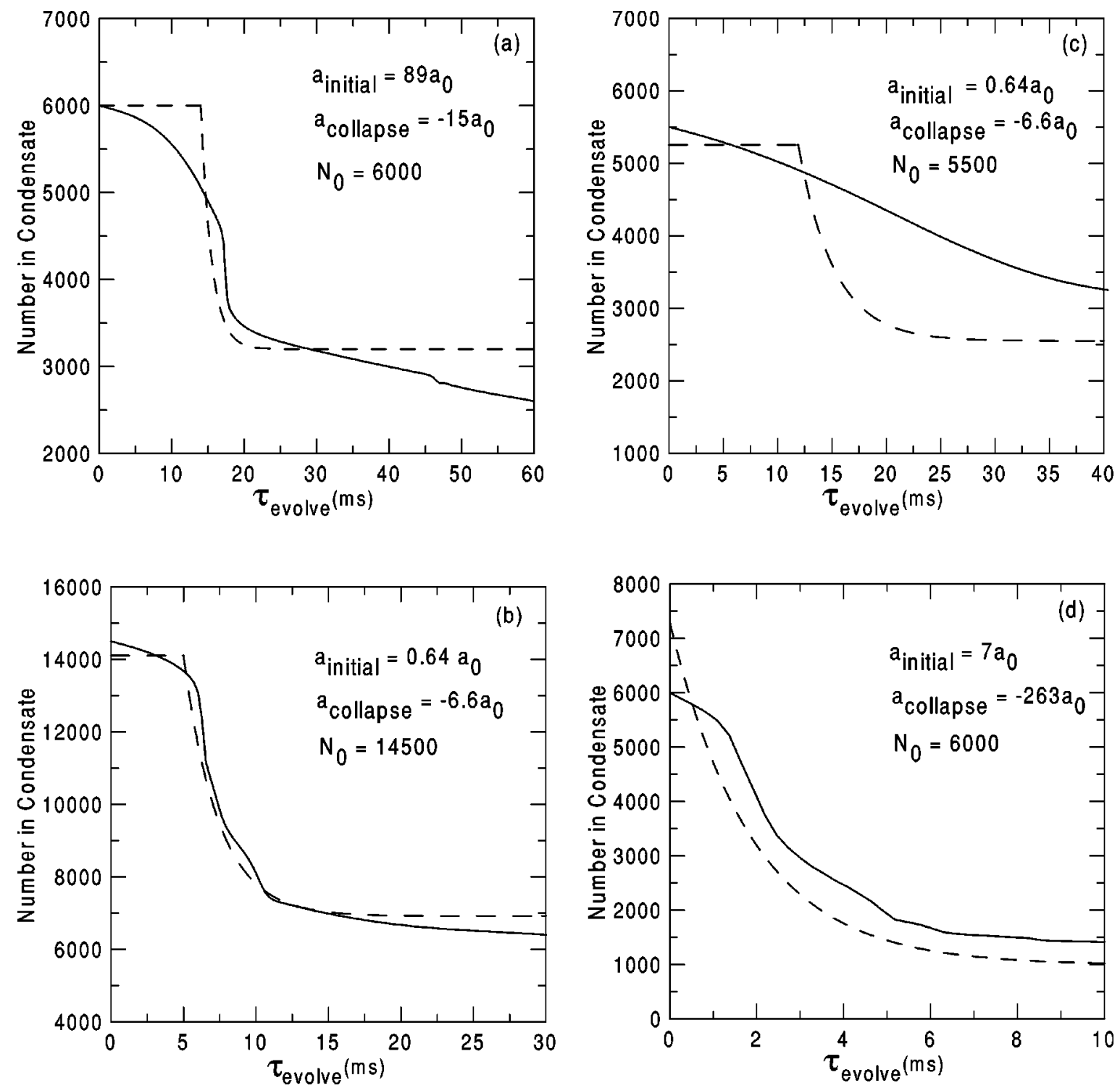

FIG. 3. More decay curves with $\xi=2$ for (a) $a_{\text {initial }}=89 a_{0}, a_{\text {collapse }}=-15 a_{0}$, and $N_{0}=6000$, (b) $a_{\text {initial }}=0.64 a_{0}, a_{\text {collapse }}=-6.6 a_{0}$, and $N_{0}=14500$, (c) $a_{\text {initial }}=0.64 a_{0}, a_{\text {collapse }}=-6.6 a_{0}$, and $N_{0}=5500$, and (d) $a_{\text {initial }}=7 a_{0}, a_{\text {collapse }}=-263 a_{0}$, and $N_{0}=6000$. Full line, present theory; dashed line, average over preliminary, unanalyzed data using Eq. (3.1) [25].

In our calculation we define $t_{\text {collapse }}$ as the time at which the spikes (irregularities), as in Fig. 2, tend to appear in the wave function. The results are plotted in Fig. 4 and compared with experimental data [14] as well as with another calculation using the mean-field GP equation in an axially symmetric trap [9]. The agreement between the two theoretical results is very good. There is also qualitative agreement between the experimental data on the one hand and the two calculations on the other hand: $t_{\text {collapse }}$ decreases with increasing $\left|a_{\text {collapse }}\right| / a_{0}$ starting from an infinite value at $\left|a_{\text {collapse }}\right|=a_{\mathrm{cr}}$ for a fixed $N_{0}$, which is $6000 \mathrm{in} \mathrm{Fig.} \mathrm{4.} \mathrm{For} \mathrm{this} N_{0}, a_{\mathrm{cr}}$ is the minimum value of $\left|a_{\text {collapse }}\right|$ that leads to the collapse and explosion. For a given $N_{0}$, a critical value of $n \equiv n_{\text {cr }}$ for collapse can be defined via $n_{\mathrm{cr}} \equiv N_{0} a_{\mathrm{cr}} / l$. As there is a discrepancy between the theoretical and experimental $n_{\text {cr }}$ for an axially symmetric trap $[12,13]$, the theoretical and experimental $a_{\mathrm{cr}}$ are also supposed to be different. The experimental $k_{\mathrm{cr}}$ $\equiv n_{\mathrm{cr}} \lambda^{1 / 6}=0.46[10]$ and the theoretical $k_{\mathrm{cr}}=0.55[12,13]$ for the axially symmetric trap used in the experiment of Donley et al. with the asymmetry parameter $\lambda=0.389$. The theoretical $a_{\text {cr }}$ should be larger than the experimental $a_{\text {cr }}$ in the same proportion. This might imply that the theoretical $t_{\text {collapse }}$ should tend to infinity for a slightly larger value of $a_{\text {collapse }}$ as in Fig. 4.

Donley et al. measured the number of remnant atoms for $a_{\text {initial }}=7 a_{0}$ and different initial number $N_{0}$ and $a_{\text {collapse }}$ and these results [26] are plotted in Figs. 5(a) and 5(b) and compared with numerical simulations performed with $\xi=3$ and 2 , respectively. The agreement is good for most cases shown in this figure. For $N_{0}=6000$, there is some discrepancy between theoretical and experimental remnant numbers. The overall agreement is better in the case with $\xi=2$ than with $\xi=3$. For $\xi=3$ the three-body recombination loss rate is larger and this leads to smaller remnant numbers compared to the case with $\xi=2$. The theoretical $N_{\mathrm{cr}}$ for a fixed negative $a_{\text {collapse }}$ is given by $N_{\mathrm{cr}}=0.55 l \lambda^{-1 / 6} /\left|a_{\text {collapse }}\right|[12,13]$. For 


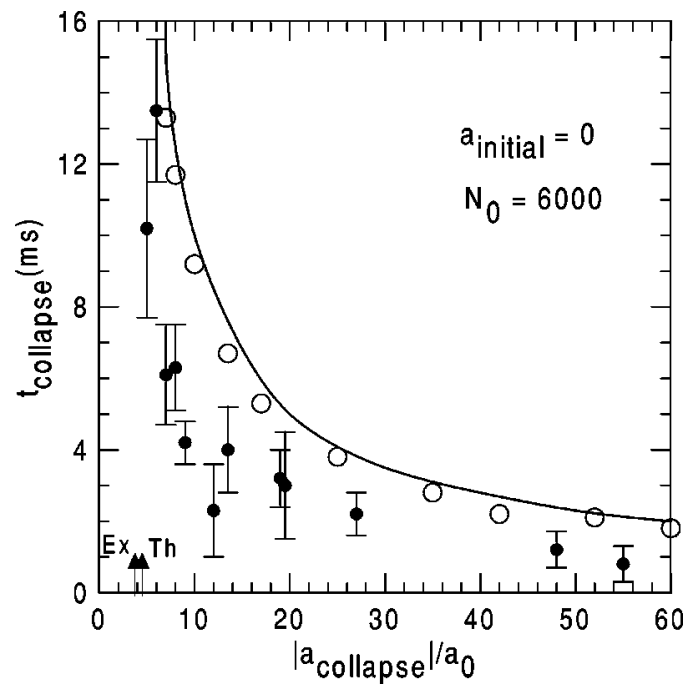

FIG. 4. The collapse time $t_{\text {collapse }}$ vs $\left|a_{\text {collapse }}\right| / a_{0}$ for $a_{\text {initial }}=0$, $N_{0}=6000$, and $\xi=2$. Solid circles with error bar, experiment [14]; open circles, axially symmetric mean-field model of Ref. [9]; arrows marked Th and Ex are theoretical (4.49) $[12,13]$ and experimental (3.75) [10] estimates of $\mid a_{\text {collapse }} / a_{0}$, respectively; full line, present theory.

$a_{\text {collapse }}=-255 a_{0},-100 a_{0},-30 a_{0}, \quad$ and $-21 a_{0}, \quad N_{\text {cr }}$ $=124,317,1057$, and 1510, respectively. Hence, from Fig. 5 we find that the number in the remnant could be much larger than $N_{\text {cr }}$ for times on the order of tens of milliseconds. However, in our simulation such a remnant continues to emit atoms at a much slower rate, and for very large times on the order of seconds the number of atoms eventually tends toward $N_{\mathrm{cr}}$.

Donley et al. observed that the remnant condensate in all cases oscillated in a highly excited collective state with approximate frequencies $2 \nu_{\text {axial }}$ and $2 \nu_{\text {radial }}$ being predominantly excited. The actual measured frequencies are 13.6(6) $\mathrm{Hz}$ and 33.4(3) Hz. To find if this behavior emerges from the present simulation we plot in Fig. 6 the sizes $x_{\text {rms }}$ and $y_{\text {rms }}$ vs time for the condensate after the jump in the scattering length to $-6.7 a_{0}$ from $7 a_{0}$ for $N_{0}=16000$. Excluding the first $20 \mathrm{~ms}$ when the remnant condensate is being formed, we find periodic oscillations in $x_{\mathrm{rms}}$ and $y_{\mathrm{rms}}$ with frequencies $13.5 \mathrm{~Hz}$ and $34 \mathrm{~Hz}$, respectively, as observed in experiment.

\section{DISCUSSION}

Although we have explained some aspects of the experiment of Donley et al., certain detailed features have not been addressed in this study. Donley et al. classified the emitted atoms in three categories: burst, missing (undetected), and jet atoms [14]. The jet atoms appear with much lower energy solely in the radial direction, possibly from the spikes in the wave function when the collapse is suddenly interrupted during the period of atom loss before the remnant is formed. Strangely enough, the emission of jet atoms is found not to possess axial symmetry always and hence it cannot be properly treated in an axially symmetric model. Moreover, a clear-cut distinction between the burst and missing atoms emitted during the explosion seems to be difficult in the
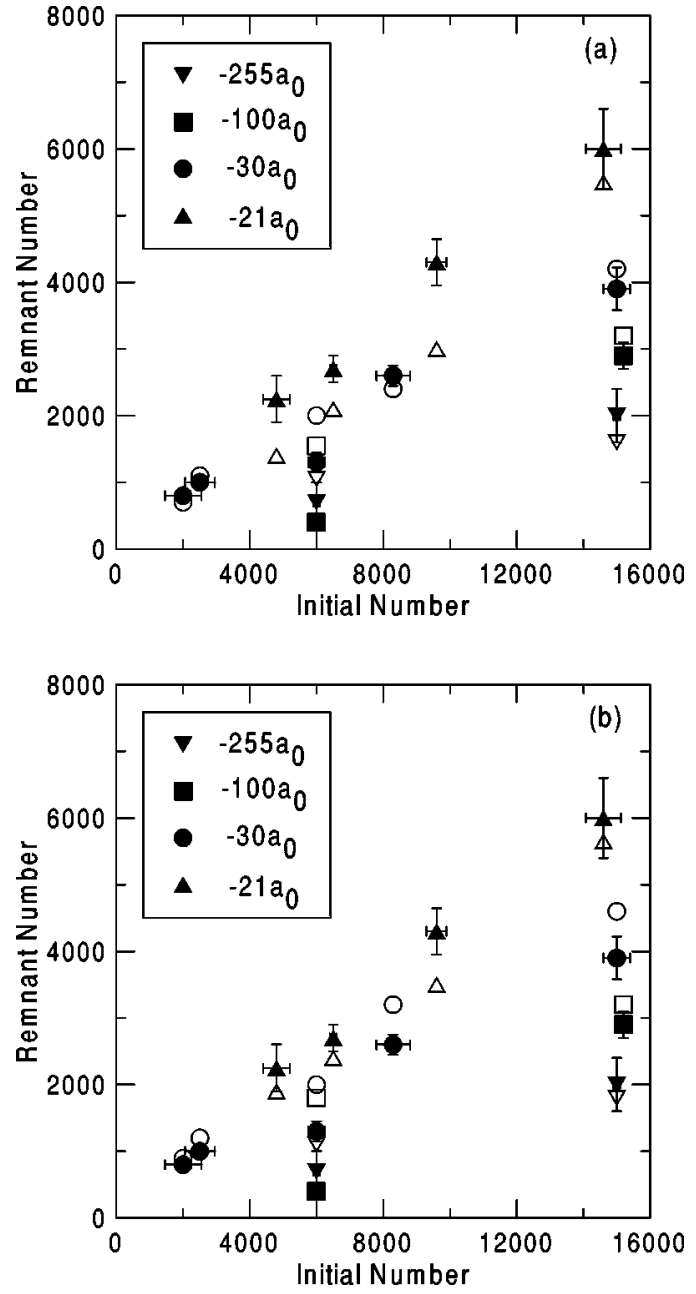

FIG. 5. Remnant number vs initial number for $a_{\text {initial }}=7 a_{0}$ and different $a_{\text {collapse }}$ for (a) $\xi=3$ and (b) $\xi=2$. The experimental results [26] with error bars are represented by solid triangles, solid circles, solid squares, and solid inverted triangles for $a_{\text {collapse }}=-21 a_{0}$, $-30 a_{0},-100 a_{0}$, and $-255 a_{0}$. The corresponding theoretical results are represented by open triangles, open circles, open squares, and open inverted triangles.

present model as, the experiment could not specify the properties (magnitude and direction of velocities) of the missing atoms. Also, because of the missing atoms it is difficult to predict the energy distribution of the burst atoms during the explosion in a mean-field analysis. Without proper identification of the missing atoms, any energy distribution calculated using the present mean-field analysis will yield the total energy of burst plus missing atoms. A careful analysis of the energy of the emitted atoms is required to explain their exclusive features and a detailed study of the wave function is needed for this purpose. Such an analysis is beyond the scope of the present investigation and would be a welcome future theoretical work.

The success of the Crank-Nicholson algorithm in alternate directions as used in this study depends on a proper discretization of the GP equation in space and time. In this study we employed a two-dimensional lattice in space of $600 \times 150$ or 90000 points $(x \leqslant 15,-30 \leqslant y \leqslant 30)$ and a time step of 0.001 . In the absence of collapse and recombination loss this dis- 


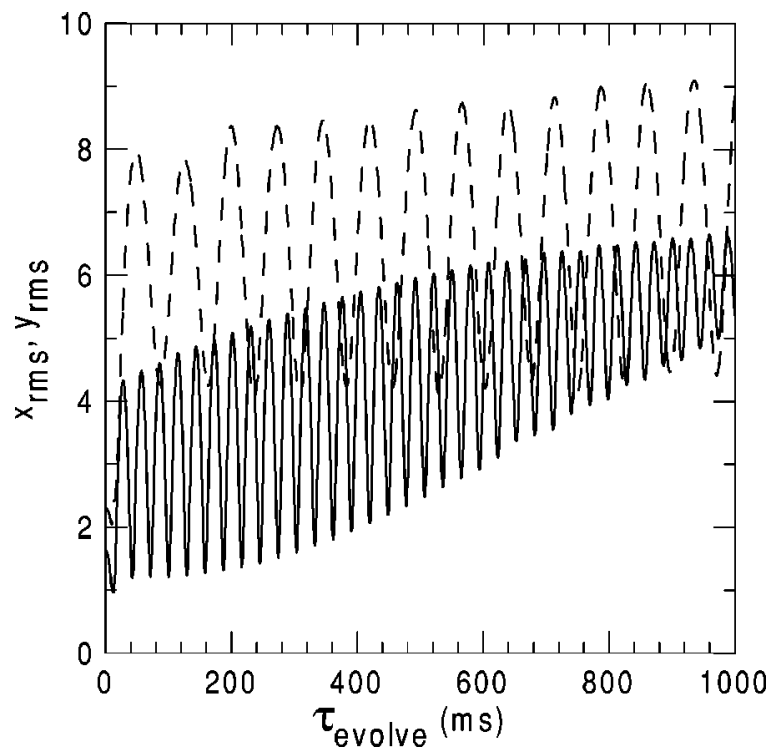

FIG. 6. The dimensionless rms sizes $x_{\text {rms }}$ (full line) and $y_{\text {rms }}$ (dashed line) expressed in units of $l / \sqrt{2}(l=26070 \AA)$ after the jump in the scattering length of a BEC of $16000{ }^{85} \mathrm{Rb}$ atoms from $a_{\text {initial }}=7 a_{0}$ to $a_{\text {collapse }}=-6.7 a_{0}$ as functions of time $\tau_{\text {evolve }}$ for $\xi$ $=2$.

cretization leads to very precise results. The accuracy is reduced in the presence of the violent collapse and explosion simulated by three-body recombination. By varying the space discretization grid and time step we found that the estimated error in the present calculation is less than $\sim 10 \%$ for time propagation up to a few tens of milliseconds.

There has been another attempt to use the mean-field GP equation in an axially symmetric trap [9] to explain the experiment of Ref. [14]. There are certain differences between the analysis of Ref. [9] and the present investigation. According to the experiment of Ref. [14], the burst atoms and missing atoms are components of expelled atoms which lose contact with the central condensate that eventually forms the remnant. Of these, the burst atoms have energy much less than the magnetic trap depth. Hence, although expelled from the central condensate they continue to be trapped and oscillate with time. The wave function of Eq. (2.3) describes only the central condensate. However, in Ref. [9] the burst atoms are considered to be the peripheral part (the spikes) of the central condensate and hence taken to be described by the mean-field Eq. (2.3). The missing atoms are actually parts of the expelled atoms that have disappeared from the trap [14]. In Ref. [9], the missing atoms have been taken to be the only component of the emitted atoms. These are the main differences between the point of view of the present analysis and that of Ref. [9].

The three-body loss rates of the two studies are also widely different. Here we employ the three-body recombination loss rate $K_{3} \simeq 9 \times 10^{-25} \mathrm{~cm}^{6} / \mathrm{s}$ for $a=-370 a_{0}$ whereas in Ref. [9] the value $K_{3} \sim 10^{-28} \mathrm{~cm}^{6} / \mathrm{s}$ was considered. In our study, for smaller values of $|a|, K_{3}$ has been scaled down using the relation $K_{3} \propto a^{2}$. The present rate is in rough agreement with the experimental rate of Ref. [16] $\left(K_{3} \sim 4.2\right.$ $\times 10^{-25} \mathrm{~cm}^{6} / \mathrm{s}$ ) and with the theoretical rate of Ref. [17]
( $K_{3} \sim 6.7 \times 10^{-25} \mathrm{~cm}^{6} / \mathrm{s}$ ) for the same value of scattering length, whereas that of Ref. [9] is orders of magnitude smaller. However, such a small three-body rate in Ref. [9] led to a large residual condensate at large time, which the authors interpreted as the sum of burst plus remnant. The use of a large three-body rate in this study led to a much smaller residual central condensate, which was identified as the remnant as in the experiment of Donley et al. [14].

However, it is reassuring to see that the $t_{\text {collapse }}$ vs $\left|a_{\text {collapse }}\right| / a_{0}$ curves of the two models in Fig. 4 agree with each other. The present calculation in Fig. 4 was performed with a nonzero loss rate $K_{3}$, whereas that in Ref. [9] was performed by setting $K_{3}=0$. We find that $K_{3}$ plays an insignificant role in this calculation at small times. Hence, the two computer routines lead to the same result in the absence of recombination loss before the beginning of the explosion.

\section{CONCLUSION}

In conclusion, we have employed a numerical simulation based on the accurate solution [13] of the mean-field GrossPitaevskii equation with a cylindrical trap to study the dynamics of the collapse and explosion as observed in the recent experiment of Donley et al. [14]. In the GP equation we include a quintic three-body nonlinear recombination loss term that accounts for the decay of the strongly attractive condensate. The results of the present simulation account for some aspects of the experiment.

In the experiment a strongly attractive ${ }^{85} \mathrm{Rb}$ condensate was prepared by ramping the scattering length to a large negative value and the subsequent decay of the collapsing and exploding condensate was measured. We have been able to understand the following features of this dynamics from the present numerical simulation. (1) The condensate undergoes collapse and explosion and finally stabilizes to a remnant condensate containing about $\sim 10 \%$ (for $\left|a_{\text {collapse }}\right|$ $>100 a_{0}$ ) to $40 \%$ (for $\left|a_{\text {collapse }}\right|<10 a_{0}$ ) of the initial number of atoms $N_{0}$ at large times. This percentage is independent of $N_{0}$ and the ramped scattering length $a_{\text {collapse }}$. The number in the remnant condensate can be much larger than the critical number for collapse $N_{\text {cr }}$ for the same atomic interaction for experimental times on the order of tens of milliseconds. (2) In both the experiment and our simulation the remnant condensate executes radial and axial oscillations in a highly excited collective state for a long time with frequencies $2 \nu_{\text {radial }}$ and $2 \nu_{\text {axial }}$. (3) After the sudden change in the scattering length to a large negative value, the condensate needs an interval of time $t_{\text {collapse }}$ before it experiences loss via explosion. Consequently, the decay starts after the interval of time $t_{\text {collapse }}$. (4) The number of atoms in the condensate decays exponentially with a decay constant $\tau_{\text {decay }}$ of a few milliseconds ( $\sim 1-3 \mathrm{~ms})$.

To conclude, a large part of the experiment on ${ }^{85} \mathrm{Rb}$ atoms of Donley et al. [14], in particular the detailed behavior of the remnant, can be understood by introducing a rather conventional three-body recombination loss in the standard 
mean-field GP equation, with a loss rate compatible with other studies $[16,17]$. The study of the detailed behavior of the burst and missing atoms and the formation of the jet in such a mean-field theory seems to be more complicated technically, but nevertheless viable, and is a subject for future investigation.

\section{ACKNOWLEDGMENTS}

The author extends sincere thanks to Dr. E. A. Donley for additional unpublished data $[25,26]$ from the experiment [14]. The work was supported in part by the CNPq and FAPESP of Brazil.
[1] M.H. Anderson, J.R. Ensher, Jr., M.R. Matthews, C.E. Wieman, and E.A. Cornell, Science 269, 198 (1995); J.R. Ensher, D.S. Jin, M.R. Matthews, C.E. Wieman, and E.A. Cornell, Phys. Rev. Lett. 77, 4984 (1996); K.B. Davis, M.O. Mewes, M.R. Andrews, N.J. van Druten, D.S. Durfee, D.M. Kurn, and W. Ketterle, ibid. 75, 3969 (1995); D.G. Fried, T.C. Killian, L. Willmann, D. Landhuis, S.C. Moss, D. Kleppner, and T.J. Greytak, ibid. 81, 3811 (1998).

[2] A. Robert, O. Sirjean, A. Browaeys, J. Poupard, S. Nowak, D. Boiron, C.I. Westbrook, and A. Aspect, Science 292, 461 (2001); F. Pereira Dos Santos, J. Léonard, J. Wang, C.J. Barrelet, F. Perales, E. Rasel, C.S. Unnikrishnan, M. Leduc, and C. Cohen-Tannoudji, Phys. Rev. Lett. 86, 3459 (2001); G. Modugno, G. Ferrari, G. Roati, R.J. Brecha, A. Simoni, and M. Inguscio, Science 294, 1320 (2001).

[3] J.M. Gerton, D. Strekalov, I. Prodan, and R.G. Hulet, Nature (London) 408, 692 (2001); C.C. Bradley, C.A. Sackett, J.J. Tollett, and R.G. Hulet, Phys. Rev. Lett. 75, 1687 (1995); C.C. Bradley, C.A. Sackett, and R.G. Hulet, ibid. 78, 985 (1997).

[4] E.P. Gross, Nuovo Cimento 20, 454 (1961); L.P. Pitaevskii, Zh. Éksp. Teor. Fiz. 40, 646 (1961) [Sov. Phys. JETP 13, 451 (1961)]; A.J. Leggett, Rev. Mod. Phys. 73, 307 (2001).

[5] F. Dalfovo, S. Giorgini, L.P. Pitaevskii, and S. Stringari, Rev. Mod. Phys. 71, 463 (1999).

[6] H. Saito and M. Ueda, Phys. Rev. A 63, 043601 (2001); Phys. Rev. Lett. 86, 1406 (2001); A. Eleftheriou and K. Huang, Phys. Rev. A 61, 043601 (2000).

[7] R.A. Duine and H.T.C. Stoof, Phys. Rev. Lett. 86, 2204 (2001).

[8] Y. Kagan, A.E. Muryshev, and G.V. Shlyapnikov, Phys. Rev. Lett. 81, 933 (1998); Y. Kagan, E.L. Surkov, and G.V. Shlyapnikov, ibid. 79, 2604 (1997); A. Gammal, T. Frederico, L. Tomio, and Ph. Chomaz, Phys. Rev. A 61, 051602 (2000); M. Ueda and K. Huang, ibid. 60, 3317 (1999).

[9] H. Saito and M. Ueda, Phys. Rev. A 65, 033624 (2002).

[10] J.L. Roberts, N.R. Claussen, S.L. Cornish, E.A. Donley, E.A. Cornell, and C.E. Wieman, Phys. Rev. Lett. 86, 4211 (2001); S.L. Cornish, N.R. Claussen, J.L. Roberts, E.A. Cornell, and C.E. Wieman, ibid. 85, 1795 (2000).

[11] R.J. Dodd, M. Edwards, C.J. Williams, C.W. Clark, M.J. Holland, P.A. Ruprecht, and K. Burnett, Phys. Rev. A 54, 661 (1996); F. Dalfovo and S. Stringari, ibid. 53, 2477 (1996); M. Houbiers and H.T.C. Stoof, ibid. 54, 5055 (1996); A. Gammal, T. Frederico, and L. Tomio, Phys. Rev. E 60, 2421 (1999).

[12] A. Gammal, T. Frederico, and L. Tomio, Phys. Rev. A 64, 055602 (2001).

[13] S.K. Adhikari, Phys. Rev. E 65, 016703 (2002); S.K. Adhikari and P. Muruganandam, J. Phys. B 35, 2831 (2002).

[14] E.A. Donley, N.R. Claussen, S.L. Cornish, J.L. Roberts, E.A. Cornell, and C.E. Wieman, Nature (London) 412, 295 (2001).

[15] S. Inouye, M.R. Andrews, J. Stenger, H.J. Miesner, D.M. Stamper-Kurn, and W. Ketterle, Nature (London) 392, 151 (1998); Ph. Courteille, R.S. Freeland, D.J. Heinzen, F.A. van Abeelen, and B.J. Verhaar, Phys. Rev. Lett. 81, 69 (1998); V. Vuletić, A.J. Kerman, C. Chin, and S. Chu, ibid. 82, 1406 (1999); E. Timmermans, P. Tommasini, M. Hussein, and A. Kerman, Phys. Rep. 315, 199 (1999).

[16] J.L. Roberts, N.R. Claussen, S.L. Cornish, and C.E. Wieman, Phys. Rev. Lett. 85, 728 (2000).

[17] B.D. Esry, C.H. Greene, and J.P. Burke, Jr., Phys. Rev. Lett. 83, 1751 (1999).

[18] S.K. Adhikari, Phys. Rev. E 62, 2937 (2000); 63, 056704 (2001); Phys. Rev. A 63, 043611 (2001); 65, 033616 (2002).

[19] M. Holland and J. Cooper, Phys. Rev. A 53, R1954 (1996); F. Dalfovo and M. Modugno, ibid. 61, 023605 (2000); P.A. Ruprecht, M.J. Holland, K. Burnett, and M. Edwards, ibid. 51, 4704 (1995); B.I. Schneider and D.L. Feder, ibid. 59, 2232 (1999); M.M. Cerimele, M.L. Chiofalo, F. Pistella, S. Succi, and M.P. Tosi, Phys. Rev. E 62, 1382 (2000).

[20] A.J. Moerdijk, H.M.J.M. Boesten, and B.J. Verhaar, Phys. Rev. A 53, 916 (1996).

[21] J.M. Gerton, C.A. Sackett, B.J. Frew, and R.G. Hulet, Phys. Rev. A 59, 1514 (1999); E.A. Burt, R.W. Ghrist, C.J. Myatt, M.J. Holland, E.A. Cornell, and C.E. Wieman, Phys. Rev. Lett. 79, 337 (1997); D.M. Stamper-Kurn, M.R. Andrews, A.P. Chikkatur, S. Inouye, H.-J. Miesner, J. Stenger, and W. Ketterle, ibid. 80, 2027 (1998).

[22] P.O. Fedichev, M.W. Reynolds, and G.V. Shlyapnikov, Phys. Rev. Lett. 77, 2921 (1996).

[23] E. Braaten and H.W. Hammer, Phys. Rev. Lett. 87, 160407 (2001); P.F. Bedaque, E. Braaten, and H.W. Hammer, ibid. 85, 908 (2000).

[24] S.E. Koonin and D.C. Meredith, Computational Physics Fortran Version (Addison-Wesley, Reading, MA, 1990), pp 169180.

[25] E.A. Donley private communication, Donley kindly made available the preliminary and unanalyzed, but nevertheless very useful unpublished data for particle loss presented in Figs. 1 and 3 (however, the data are not shown in these figures), and checked that the results of our numerical simulation for all the cases presented in these figures agree well with the unpublished data.

[26] E.A. Donley private communication; E.A. Donley, N.R. Claussen, S.L. Cornish, J.L. Roberts, E.A. Cornell, and C.E. Wieman (unpublished). 